\title{
Utilizing Blockchain for Electronic Wellbeing Records
}

\author{
Sugeng Widada ${ }^{1}$, Warseno ${ }^{2}$, Sugina ${ }^{3}$, Sulthan Taqi Sampoerna ${ }^{4}$ \\ Universitas Raharja, \\ Modern, Jl. Jenderal Sudirman No.40, RT.002/RW.006, Cikokol, Kec. Tangerang, Kota Tangerang, \\ Banten 15117Country1 \\ e-mail: sugeng.widada@raharja.info ${ }^{1}$, warseno@raharia.info ${ }^{2}$, \\ sugina@raharia.info ${ }^{3}$, sulthan@ @raharja.info $^{4}$
}

Widada, S., Warseno, Sugina, Sampoerna T.S. Utilizing Blockchain for Electronic Wellbeing Records

Blockchain Frontier Technology (B-Front), 1(2), 11-22.

DOI : https://journal.pandawan.id/b-front/article/view/47

\begin{abstract}
Blockchain has been an interesting area of exploration for some time and the advantages it provides have been exploited by a variety of different businesses. Basically, the medical service area has benefited greatly from blockchain innovations due to safety, security, confidentiality and decentralization. Overall, the Electronic Wellbeing Record (EHR) system addresses issues related to information security, honesty and fairness. In this paper, we talk about how blockchain innovation can be used to change the EHR framework and can be the answer to this problem. We present a system that can be used for the implementation of blockchain innovations in the field of medical services for EHR. The essence of our proposed system is first and foremost to implement blockchain innovations for EHR and in addition to provide secure electronic records capacity by characterizing granular access rules for clients of the proposed structure. Moreover, this structure also speaks to the flexibility issue that blockchain innovation sees as a whole through the use of the off-chain capacity of the record. This structure gives the EHR framework the advantage of having a blockchain-based setup that is versatile, secure, and basic. The information on the blockchain is apparent to everybody that is available on the chain this makes the information weak which is anything but an ideal result for a decentralized stage.
\end{abstract}

Keywords: Blockchain, health records, electronic health records, decentralization

\section{Introduction}

The new approach in innovation is influencing all pieces of human existence and is changing the manner in which we utilize and see things beforehand. Actually, like the progressions innovation has presented in different areas of life, it is additionally tracking down new ways for development in the medical care area[1]. The primary advantages that progression in innovation is offering are to further develop security, client experience and different parts of the medical care area. These advantages were presented by Electronic Wellbeing Record $(E H R)$ and Electronic Clinical Record (EMR) frameworks[2]. Notwithstanding, they actually face

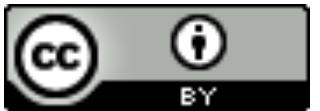

Copyright (c) 2022 Widada, S., Warseno, Sugina, Sampoerna T.S. (Author). This work is licensed under a Creative Commons Attribution 4.0 
a few issues in regards to the security of clinical records, client responsibility for, information trustworthiness and so forth. The answer for these issues could be the utilization of an original innovation, i.e., Blockchain[3]. This innovation offers to give a safe, carefully designed stage for putting away clinical records and other medical care related data. Prior to the coming of current innovation, the medical care area utilized paper based frameworks to store the clinical records, i.e., utilizing manually written components[4]. This paper-based clinical record framework was wasteful, shaky, chaotic and was not carefully designed. It additionally confronted the issue of information duplication[5]. Also excess as every one of the foundations that patient visited had different duplicates of the patient's clinical records. The medical care area confronted a pattern shift towards EHR frameworks that were intended to join paper-based and electronic clinical records (EMR)[6]. These frameworks were utilized to store clinical notes and the research facility brings about its numerous parts. They were proposed to upgrade the wellbeing part of the patients by forestalling blunders and expanding data access[7]. The objective of EHR frameworks was to tackle the issues looked by the paper-based medical care records and to give an effective framework that would change the condition of the medical care area[8]. The EHR frameworks have been executed in various emergency clinics all over the planet due the advantages it gives, basically the improvement in security and its expense viability[9]. They are viewed as an imperative piece of the medical services area as it gives a lot of usefulness to medical services[10]. These functionalities are electronic capacity of clinical records, patients' arrangement of the board, charging and records, and lab tests. They are accessible in a considerable lot of the EHR frameworks being utilized in the medical care area[11]. The essential center is to give secure, temper-evidence, and shareable clinical records across various stages. Notwithstanding the way that the thought behind utilization of EHR frameworks in the clinics or medical services was to work on the nature of medical care, and didn't meet the assumptions related with them. A review was led in Finland to track down the encounters of nursing staff with the EHR, it was reasoned that EHR frameworks dealt with the issues identified with them being temperamental and having a helpless condition of ease of use[12]. The EHR framework likewise deals with some different issues which are as per the following:

\section{A. INTEROPERABILITY}

It is the way for various data frameworks to trade data between them. The data ought to be replaceable and should be usable for additional reasons. A significant part of EHR frameworks is its Well Being Data Trade (HIE) or overall information sharing angle[13]. With various EHR frameworks being sent in different emergency clinics they have a fluctuating degree of phrasings, specialized and useful abilities which makes it to have no all around characterized standard. In addition, at specialized level the clinical records being traded ought to be interpretable, and that deciphered snippet of data could be additionally utilized[14].

\section{B. INFORMATION ASYMMETRY}

Today the best issue in the medical services area characterized by the pundits is data imbalance which alludes to one party having preferable admittance to data over the other party[15]. On account of EHR frameworks, or as a general rule, the medical services area is experiencing this issue as specialists or emergency clinics approach the patient's records, along these lines making it focal[16]. Assuming a patient needs to get to his clinical records he would need to follow a long and dreary interaction to get to them. The data is brought together to just a solitary medical care association and its control is simply given to the clinics or associations[17].

\section{DATA BREACHES}

Information breaks in the medical services area likewise require the need of a superior stage. A review was finished examining the information breaks in EHR frameworks and it portrayed that 173 million information passages have been compromised in these frameworks 
since October 2009[18]. One more review directed by Argaw et al, clarifies that emergency clinics have turned into an objective of digital assaults and an expanding pattern has been seen by the scientists while leading this review that a ton of exploration work has been done in this area[19]. Additionally, numerous EHR frameworks are not intended to satisfy the necessities and prerequisites of the patients and face the issues identified with shortcoming and helpless transformation of these frameworks. The writing likewise recommends that utilization of EHRs have acquainted adverse results with data handling. These issues make it sensible to find a stage that would be useful in changing the medical serv

ices area to show restraint focused, i.e., Blockchain. A stage which is secure, straightforward and it additionally gives information uprightness to the clinical records of the patients. This paper proposes a structure that makes such a decentralized stage that would store patient's clinical records and give access of those records to suppliers or concerned people, i.e., patients[20]. We additionally expect to take care of the adaptability issue of blockchain, as it isn't in the plan of blockchain to store gigantic volumes of information on it. In this way, we would utilize an off-chain scaling strategy that utilizes the fundamental medium to tackle the adaptability issue by putting away the information on that medium. Besides, our proposed work is planning to tackle the previously mentioned data deviation and information breaks issue looked at by the EHR framework[21].

\section{Literature review}

\section{Blockchain Foundation}

Initially intended for keeping a monetary record, the blockchain worldview can be reached out to give a summed up structure to carrying out decentralized figure assets. Each process asset can be considered as a singleton state-machine that can change between states through cryptographically-got exchanges[22]. While creating another state-machine, the hubs encode rationale which characterizes substantial state advances and transfer it onto the blockchain. From that point on, the squares diary a progression of legitimate exchanges that, when steadily executed with the state from the past block, transform the state-machine into its present status[23]. The Proof of Work agreement calculation and its fundamental distributed convention secure the express machines' state and progressing rationale from altering, and furthermore share this data with all hubs partaking in the framework. Hubs can subsequently question the state machines whenever and get an outcome which is acknowledged by the whole organization with high sureness[24]. This exchange based state-machine speculation of the blockchain is casually alluded to as brilliant agreements. Ethereum is quick to endeavor a full execution of this thought. It incorporates into the blockchain a Turing-complete guidance set to permit brilliant agreement programming and a capacity ability to oblige on-chain state. We respect the adaptability of its programming language as a significant property with regards to EHR the board. This property can empower progressed usefulness (multi-party assertion, offering, notoriety, and so on) to be coded into our proposed framework, adjusting to follow contrasts in guideline and changes in partners' needs[25]. We use Ethereum's shrewd agreements to make astute portrayals of existing clinical records that are put away inside individual hubs of the organization. We develop the agreements to contain metadata about the record proprietorship, consents and information uprightness. The blockchain exchanges in our framework convey cryptographically marked directions to deal with these properties. The agreement's state transition capacities complete arrangements, authorizing information variation simply by real exchanges. Such strategies can be intended to carry out any arrangement of rules which oversee a specific clinical record, as long as it very well may be addressed computationally. For instance, a strategy might authorize that different exchanges addressing assent are sent from the two patients and care suppliers, prior to giving review 
consents to an outsider[26]. To explore the conceivably huge measure of record portrayals, our framework structures them on the blockchain by carrying out three sorts of agreements[27].

Execution Modes: Execution happens as a call or a "sendTransaction" where a call is upset on the neighborhood machine of the end-client.

It is a speedy strategy, however it deals with a read-just worldview[28]. They are never sent to the organization and thus need not be verified for intra network correspondence. Exchanges are sent utilizing "sendTransaction", which are sought after by confirmed diggers, with a capability of radically affecting the chain.

Communication Methods: There are two manners by which Ethereum agreements might seek after intra and bury network correspondence[29]. First is by means of Messages, which are among contracts and are not impacted by the mining that happens on the blockchain. Exchanges begin from the end-clients which start different cycles[30].

\section{Constituent Nodes}

The separation not just builds the versatility of the Blockchain to such an extent that different partners can connect with the chain to benefit from assets in a proficient way[31]. Hubs establishing the square chain are isolated into three classes:

- Full Nodes: Each exchange is put away, with each individual square from the blockchain. A bigger extra room and expanded computational power is required.

- Light Nodes: Just square headers are put away to confirm the adjustment of any square or an exchange that happened. Explicit information can be gotten through the light hubs.

- Archive Nodes: Store each exchange, each square and the receipts of the exchanges that happen. This empowers the organization to recover required information.

\section{IT Components}

1) Database Manager: A supervisor explores among the data sets that save the patient wellbeing records and connection age to highlight the records. The supervisor will likewise make hashes of the records and questions created for the connection. Uprightness is saved utilizing the hashes as they keep away from direct access of the data set.

2) Cipher Manager: The cryptographic necessities of the worldview will be dealt with by the Code Supervisor. Encryption-unscrambling of the records that are put away/traded will be attempted by the Chief. Keys are utilized for this reason; symmetric key style and public key style encryption pattern are used.

\section{Research Methods \\ Blockchain Technology}

This innovation was presented by Nakamoto, for his famous work of advanced cash or digital currency, i.e., bitcoin. Nakamoto utilized blockchain innovation to tackle the twofold spending issue of bitcoin yet soon this clever innovation was being utilized in numerous different applications. Blockchain is a chain of squares that are associated together and are consistently developing by putting away exchanges on the squares. This stage utilizes a decentralized methodology that permits the data to be disseminated and that each piece of appropriated data or ordinarily known as information have shared possession. Blockchains hold bunches of exchanges that are hashed subsequently giving them security and they are overseen by distributed organizations. A blockchain has specific advantages like security, namelessness, and respectability of information with no outsider intercession. These advantages settle on a 

sensible decision to store patient's clinical records on it, on the grounds that the development of innovation in the medical services industry has made the security of patient's clinical information a main concern. Various specialists have additionally recognized that utilizing blockchain innovation in medical services would be an attainable arrangement

\section{A. Design}

To comprehend blockchain engineering let us utilize the accompanying figure 1 that clarifies the entire course of an exchange being sent from a client on the blockchain network.

1. Another exchange being sent by a client on the blockchain network proposes that another square is made. A square in the blockchain is utilized for keeping exchanges in them and these squares are disseminated to every one of the associated hubs in the organization. That exchange put inside a square is communicated to every one of the hubs in the organization. Every one of the hubs in the organization have a duplicate of the total blockchain that helps them in the confirmation process. At the point when a square containing the client exchange is communicated to the associated hubs as a whole, they confirm that the square isn't altered using any and all means. Assuming this confirmation brings about progress then the hubs add that square in their own duplicate of blockchain

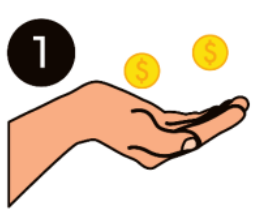

client sends exchange
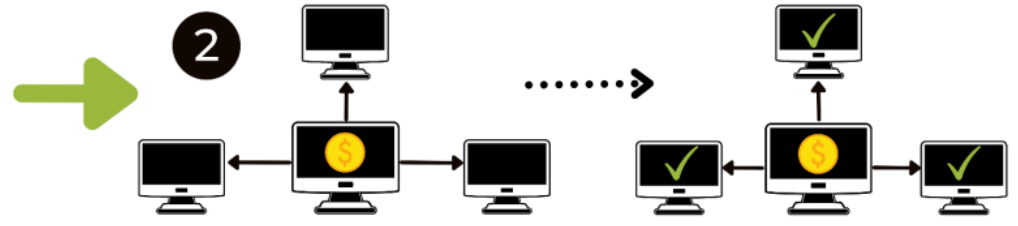

exchange is shipped off every one of the associated hubs on a p2p network for approval

associated hubs perform approval on the exchange by utilizing known agreement algoritma

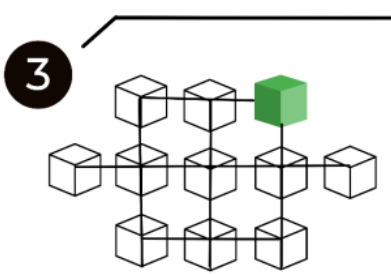

at the point when exchange is approved the square is added to the blockchain
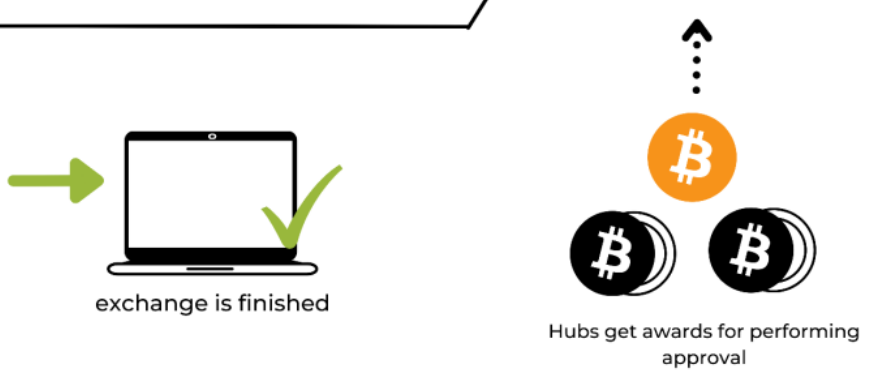

Fig. 1 An overview of blockchain architecture.

2. This entire course of the square being added on the blockchain is finished by the hubs coming upon an agreement where they conclude which squares are substantial to be added on the blockchain and which are not. This approval is performed by the associated hubs utilizing some known calculations to confirm the exchange and to guarantee that the sender is a verified piece of the organization. At the point when a hub prevails with regards to playing out the approval that hub is compensated with cryptographic money. This course of approving the exchange is known as mining and the hub playing out this approval is known as digger

3. Later approval is done that square is added to the blockchain.

4. Later the entire course of approval is played out and the exchange is finished. 

Some fundamental ideas of blockchain innovation can be perceived in the accompanying depictions

\section{B. Block}

As clarified before blockchain are shaped together by various squares associated together in a distributed organization in this way making a decentralized application. The header of these squares contains hashes of past blocks in them. A square contains three things in it which are information, hash of the current square and a hash of a past block. The information could be anything as it relies upon the kind of blockchain. As if there should arise an occurrence of bitcoin, the information comprises coins that are really electronic money. The hash that is put away in these squares contains a SHA-256 cryptographic calculation which is utilized for novel distinguishing proof of a square on the chain.

\section{Agreement Calculation}

Each square that is added on the chain would have to adhere to some agreement guidelines for it to be added on the blockchain. For this reason blockchain innovation utilizes agreement calculations. The most widely recognized agreement calculation utilized is Proof of Work (PoW) calculation and it was utilized by Nakamoto, in the bitcoin network. The fundamental working of this calculation is that there are a number of hubs or members on a blockchain network so when an exchange is mentioned to be added on the organization by any participating hub it should be determined. This cycle is called mining and the hubs that are playing out these estimations are excavators.

\section{Key Highlights Of Blockchain}

1) Decentralization

With blockchain the data is disseminated across the organization rather than at one main issue. This likewise makes the control of data to be conveyed and dealt with by agreement came to upon by shared contribution from the hubs associated with the organization. The information that was before assembled at one main issue is presently taken care of by many confided in substances.

\section{2) Data Transparency}

Accomplishing information straightforwardness in any innovation is to have a trust based connection between elements. The information or record in question ought to be gotten and temper evidence. Any information being put away on the blockchain isn't aggregated at one spot and isn't constrained by one hub yet is rather circulated across the organization. The responsibility is currently shared and this makes it to be straightforward and secure from any outsider mediation.

\section{3) Security and Privacy}

Blockchain innovation utilizes cryptographic capacities to give security to the hubs associated with its organization. It utilizes SHA-256 cryptographic calculation on the hashes that are put away on the squares. SHA represents Secure Hashing Algorithm, these hashes give security to the blockchain as information honesty is guaranteed by them. Cryptographic hashes are solid one way works that create checksum for computerized information that can't be utilized for information extraction. This makes blockchain as such a decentralized stage made secure by the cryptographic methodologies which makes it to be a decent choice for security assurance of specific applications. 



\section{E. Challenges Looked by Blockchain Innovation}

1) Scalability and Storage Capacity

Capacity of information on the blockchain creates two primary issues, i.e., secrecy and adaptability. The information on the blockchain is apparent to everybody that is available on the chain this makes the information weak which is anything but an ideal result for a decentralized stage. The information put away on the blockchain would contain patient clinical history, records, lab results, X-beams reports, X-ray results and numerous different reports, all of this voluminous information is to be put away on the blockchain that would exceptionally influence the capacity limit of blockchain.

\section{2) Lack of Social Skills}

The way blockchain innovation works is justifiable by not many individuals. This innovation is as yet in its underlying stages and is continually advancing. Additionally, the shift from confided in EHR frameworks to the blockchain innovation would take time as medical clinics, or some other medical services establishments need to totally shift their frameworks to blockchain.

3) Lack of Universally Defined Standards

As this innovation is as yet in the underlying stages and is continually advancing so there is no characterized standard for it. Due to this the execution of this innovation in the medical care area would likewise take additional time and exertion. As it would require principles from worldwide specialists that ignore the normalization cycle of any innovation. These all inclusive guidelines would benefit in choosing the information size, information organization and sort of information that could be put away on the blockchain. Besides, the transformation of this innovation would become more straightforward because of the characterized principles, as they could be handily authorized in the associations.

\section{Results and discussion Starters}

This segment officially depicts the primers utilized in the proposed system. It portrays the product stage utilized for improvement of this system and its benefits. Ethereum and IPFS being the most noticeable and significant for execution of this system are likewise examined in the accompanying segment.

\section{A. Ethereum}

Ethereum is a conveyed blockchain network that utilizes the possibility of blockchain that was recently utilized in the famous digital currency Bitcoin. Ethereum was officially presented in 2015 and the thought behind Ethereum was to make a trustless shrewd agreement stage that would be open-source and would likewise hold the element of programmable blockchain. This innovation likewise shares the shared systems administration that makes it appropriated. This stage additionally utilizes its own digital money known as Ethers. This digital money can be utilized for dividing it among accounts associated on Ethereum blockchain. Ethereum additionally gives the developers a language in which they can redo their own blockchain, this language is known as Robustness. It was produced for brilliant agreements that are the principle element of Ethereum. 



\section{B. Data Exchange}

In Ethereum, exchange is the manner in which outer substance would interface with Ethereum. It tends to be utilized by the outer client to refresh the condition of the record or data put away on the Ethereum blockchain network. An Ethereum exchange contains following components:

- From - message sender, having a 20-bytes address.

- To - message beneficiary, likewise having a 20-bytes address.

- Value - the asset sum (wei) moved from sender to beneficiary

- Data (optional) - contains the message that is being shipped off the beneficiary

- Gas - For each exchange on the Ethereum blockchain the sender needs to pay a few expenses for playing out that activity this charge is known as Gas. Each exchange contains as far as possible and gas cost in it

- Gas Price: that expense the exchange sender will pay for gas

- Gas Limit: greatest gas that could be paid for this exchange

\section{Savvy Agreements}

Savvy contracts are known as the piece of code that is utilized to play out any assignment on the blockchain. This piece of code is executed when the clients send the exchanges. They run on the blockchain straightforwardly in this manner making themselves secure from any sort of altering and changes. Savvy contracts usually use strong language and they can be utilized to program any sort of activity that a software engineer needs to do on the blockchain. Subsequent to programming the necessary activities the software engineers can order them by utilizing EVM bytecode that would be clarified in the next area. Also in the wake of assembling them it may very well be executed and conveyed on the Ethereum blockchain. The programming language of JavaScript and Python are embodied with the Strength language given by Ethereum to compose code in savvy contracts.

\section{Ethereum Virtual Machine}

The key advantages that Ethereum stage offers incorporate the programmable blockchain. It gives its clients the decision to make their own applications working on the Ethereum. The applications assembled utilizing this stage are known asDistributed Applications(DApps). They contain various conventions that are bundled together to make a stage for DApps. These DApps contain savvy gets that have code characterized by the client to play out some characterized undertaking of an application. That code is sent and executed utilizing the Ethereum Virtual Machine (EVM). In this way, the applications that are made utilizing the savvy contract are in reality being run on EVM.

E. Interplanetary File System (IPFS)

IPFS is a convention that utilizes shared organization for information stockpiling. It gives secure information stockpiling as information put away on IPFS is shielded from any modification. It utilizes a cryptographic identifier that shields the information from adjustment as any endeavor to make change on the information put away on IPFS must be finished by changing the identifier. Every one of the information documents put away on IPFS contains a hash esteem that is produced cryptographically. It is remarkable and is utilized for recognizable proof of put away information record on the IPFS. This solid stockpiling methodology of IPFS convention settles on it a good decision for putting away basic and touchy information. The cryptographic hash that is produced could be put away on the decentralized application to lessen the comprehensive computational activities over the blockchain. IPFS convention works utilizing a distributed (P2P) organization, this organization contains an information structure known as IPFS object tha 

contains information and connection in it. Information is unstructured parallel information and connection comprises a cluster. The IPFS convention works in the accompanying manner:

- Documents put away on IPFS are appointed an interesting cryptographic hash

- Copy records are not permitted to exist on the IPFS organization

- A hub on the organization stores content and record data of the hub

\section{Conclusion}

This innovation offers to give a safe, carefully designed stage for putting away clinical records and other medical care related data. Prior to the coming of current innovation, the medical care area utilized paper based frameworks to store the clinical records, i.e., utilizing manually written components. The objective of EHR frameworks was to tackle the issues looked by the paper-based medical care records and to give an effective framework that would change the condition of the medical care area. Notwithstanding the way that the thought behind utilization of EHR frameworks in the clinics or medical services was to work on the nature of medical care, and didn't meet the assumptions related with them. On account of EHR frameworks, or as a general rule, the medical services area is experiencing this issue as specialists or emergency clinics approach the patient's records, along these lines making it focal. A stage which is secure, straightforward and it additionally gives information uprightness to the clinical records of the patients. In this way, we would utilize an off-chain scaling strategy that utilizes the fundamental medium to tackle the adaptability issue by putting away the information on that medium. Literature review Blockchain Foundation Initially intended for keeping a monetary record, the blockchain worldview can be reached out to give a summed up structure to carrying out decentralized figure assets. Hubs establishing the square chain are isolated into three classes: Full Nodes: Each exchange is put away, with each individual square from the blockchain. Nakamoto utilized blockchain innovation to tackle the twofold spending issue of bitcoin yet soon this clever innovation was being utilized in numerous different applications. Blockchain is a chain of squares that are associated together and are consistently developing by putting away exchanges on the squares.

These advantages settle on a sensible decision to store patient's clinical records on it, on the grounds that the development of innovation in the medical services industry has made the security of patient's clinical information a main concern. Various specialists have additionally recognized that utilizing blockchain innovation in medical services would be an attainable arrangement. To comprehend blockchain engineering let us utilize the accompanying figure 1 that clarifies the entire course of an exchange being sent from a client on the blockchain network. A square in the blockchain is utilized for keeping exchanges in them and these squares are disseminated to every one of the associated hubs in the organization. This entire course of the square being added on the blockchain is finished by the hubs coming upon an agreement where they conclude which squares are substantial to be added on the blockchain and which are not. This course of approving the exchange is known as mining and the hub playing out this approval is known as digger Later approval is done that square is added to the blockchain. For this reason blockchain innovation utilizes agreement calculations. The fundamental working of this calculation is that there are a number of hubs or members on a blockchain network so when an exchange is mentioned to be added on the organization by any participating hub it should be determined. Key Highlights Of Blockchain Decentralization With blockchain the data is disseminated across the organization rather than at one main issue. Any information being put away on the blockchain isn't aggregated at one spot and isn't constrained by one hub yet is rather circulated across the organization. Security and Privacy Blockchain innovation utilizes cryptographic capacities to give security to the hubs associated with its organization. Challenges 
Looked by Blockchain Innovation Scalability and Storage Capacity Capacity of information on the blockchain creates two primary issues, i.e., secrecy and adaptability.

The information on the blockchain is apparent to everybody that is available on the chain this makes the information weak which is anything but an ideal result for a decentralized stage. The information put away on the blockchain would contain patient clinical history, records, lab results, X-beams reports, X-ray results and numerous different reports, all of this voluminous information is to be put away on the blockchain that would exceptionally influence the capacity limit of blockchain. Additionally, the shift from confided in EHR frameworks to the blockchain innovation would take time as medical clinics, or some other medical services establishments need to totally shift their frameworks to blockchain. These all inclusive guidelines would benefit in choosing the information size, information organization and sort of information that could be put away on the blockchain. Ethereum is a conveyed blockchain network that utilizes the possibility of blockchain that was recently utilized in the famous digital currency Bitcoin. Ethereum was officially presented in 2015 and the thought behind Ethereum was to make a trustless shrewd agreement stage that would be open-source and would likewise hold the element of programmable blockchain. It tends to be utilized by the outer client to refresh the condition of the record or data put away on the Ethereum blockchain network. IPFS convention works utilizing a distributed (P2P) organization, this organization contains an information structure known as IPFS object that contains information and connection in it. The IPFS convention works in the accompanying manner: Documents put away on IPFS are appointed an interesting cryptographic hash Copy records are not permitted to exist on the IPFS organization A hub on the organization stores content and record data of the hub

\section{References}

[1] S. Alkhushayni, D. Al-Zaleq, and N. Kengne, "Blockchain technology applied to electronic health records," in Proceedings of 32nd International Conference on, 2019, vol. 63, pp. 34-42.

[2] A. S. Anwar, U. Rahardja, A. G. Prawiyogi, and N. P. L. Santoso, "iLearning Model Approach in Creating Blockchain Based Higher Education Trust," Int. J. Artif. Intell. Res., vol. 6 , no. 1, 2022.

[3] J. H. Beinke, C. Fitte, and F. Teuteberg, "Towards a stakeholder-oriented blockchainbased architecture for electronic health records: design science research study," J. Med. Internet Res., vol. 21, no. 10, p. e13585, 2019.

[4] U. Chelladurai and S. Pandian, "A novel blockchain based electronic health record automation system for healthcare," J. Ambient Intell. Humaniz. Comput., pp. 1-11, 2021.

[5] L. Chen, W.-K. Lee, C.-C. Chang, K.-K. R. Choo, and N. Zhang, "Blockchain based searchable encryption for electronic health record sharing," Futur. Gener. Comput. Syst., vol. 95, pp. 420-429, 2019.

[6] S. Chenthara, K. Ahmed, H. Wang, F. Whittaker, and Z. Chen, "Healthchain: A novel framework on privacy preservation of electronic health records using blockchain technology," PLoS One, vol. 15, no. 12, p. e0243043, 2020.

[7] G. G. Dagher, J. Mohler, M. Milojkovic, and P. B. Marella, "Ancile: Privacy-preserving framework for access control and interoperability of electronic health records using blockchain technology," Sustain. cities Soc., vol. 39, pp. 283-297, 2018.

[8] A. Dubovitskaya et al., "ACTION-EHR: Patient-centric blockchain-based electronic health record data management for cancer care," J. Med. Internet Res., vol. 22, no. 8, p. e13598, 2020.

[9] A. Ekblaw, A. Azaria, J. D. Halamka, and A. Lippman, "A Case Study for Blockchain in Healthcare:'MedRec' prototype for electronic health records and medical research data," in Proceedings of IEEE open \& big data conference, 2016, vol. 13, p. 13.

[10] H. Guo, W. Li, M. Nejad, and C.-C. Shen, "Access control for electronic health records with hybrid blockchain-edge architecture," in 2019 IEEE International Conference on Blockchain (Blockchain), 2019, pp. 44-51. 
[11] R. Guo, H. Shi, Q. Zhao, and D. Zheng, "Secure attribute-based signature scheme with multiple authorities for blockchain in electronic health records systems," IEEE access, vol. 6, pp. 11676-11686, 2018.

[12] M. Kassab, J. DeFranco, T. Malas, V. V. G. Neto, and G. Destefanis, "Blockchain: a panacea for electronic health records?," in 2019 IEEE/ACM 1st International Workshop on Software Engineering for Healthcare (SEH), 2019, pp. 21-24.

[13] B. Mardisentosa, U. Rahardja, K. Zelina, F. P. Oganda, and M. Hardini, "Sustainable Learning Micro-Credential using Blockchain for Student Achievement Records," in 2021 Sixth International Conference on Informatics and Computing (ICIC), 2021, pp. 1-6.

[14] A. H. Mayer, C. A. da Costa, and R. da R. Righi, "Electronic health records in a blockchain: a systematic review," Health Informatics J., vol. 26, no. 2, pp. 1273-1288, 2020.

[15] R. N. Nortey, L. Yue, P. R. Agdedanu, and M. Adjeisah, "Privacy module for distributed electronic health records (EHRs) using the blockchain," in 2019 IEEE 4th International Conference on Big Data Analytics (ICBDA), 2019, pp. 369-374.

[16] J. Passerat-Palmbach et al., "Blockchain-orchestrated machine learning for privacy preserving federated learning in electronic health data," in 2020 IEEE International Conference on Blockchain (Blockchain), 2020, pp. 550-555.

[17] M. T. Quasim, A. A. E. Radwan, G. M. M. Alshmrani, and M. Meraj, "A Blockchain Framework for Secure Electronic Health Records in Healthcare Industry," in 2020 International Conference on Smart Technologies in Computing, Electrical and Electronics (ICSTCEE), 2020, pp. 605-609.

[18] B. L. Radhakrishnan, A. S. Joseph, and S. Sudhakar, "Securing blockchain based electronic health record using multilevel authentication," in 2019 5th International Conference on Advanced Computing \& Communication Systems (ICACCS), 2019, pp. 699-703.

[19] U. Rahardja, Q. Aini, F. P. Oganda, and V. T. Devana, "Secure Framework Based on Blockchain for E-Learning During COVID-19," in 2021 9th International Conference on Cyber and IT Service Management (CITSM), 2021, pp. 1-7.

[20] M. S. Rahman, I. Khalil, P. C. Mahawaga Arachchige, A. Bouras, and X. Yi, "A novel architecture for tamper proof electronic health record management system using blockchain wrapper," in Proceedings of the 2019 ACM International Symposium on Blockchain and Secure Critical Infrastructure, 2019, pp. 97-105.

[21] S. Ramachandran, O. O. Kiruthika, A. Ramasamy, R. Vanaja, and S. Mukherjee, "A Review on Blockchain-Based Strategies for Management of Electronic Health Records (EHRs)," in 2020 International Conference on Smart Electronics and Communication (ICOSEC), 2020, pp. 341-346.

[22] P. P. Ray, B. Chowhan, N. Kumar, and A. Almogren, "BloTHR: Electronic Health Record Servicing Scheme in loT-Blockchain Ecosystem," IEEE Internet Things J., 2021.

[23] F. A. Reegu, S. Mohd, Z. Hakami, K. K. Reegu, and S. Alam, "Towards Trustworthiness of Electronic Health Record system using Blockchain," Ann. Rom. Soc. Cell Biol., vol. 25, no. 6, pp. 2425-2434, 2021.

[24] A. Shahnaz, U. Qamar, and A. Khalid, "Using blockchain for electronic health records," IEEE Access, vol. 7, pp. 147782-147795, 2019.

[25] S. Shi, D. He, L. Li, N. Kumar, M. K. Khan, and K.-K. R. Choo, "Applications of blockchain in ensuring the security and privacy of electronic health record systems: A survey," Comput. Secur., p. 101966, 2020.

[26] M. Shuaib, S. Alam, M. S. Alam, and M. S. Nasir, "Compliance with HIPAA and GDPR in blockchain-based electronic health record," Mater. Today Proc., 2021.

[27] F. Tang, S. Ma, Y. Xiang, and C. Lin, "An efficient authentication scheme for blockchainbased electronic health records," IEEE access, vol. 7, pp. 41678-41689, 2019.

[28] J. Vora et al., "BHEEM: A blockchain-based framework for securing electronic health records," in 2018 IEEE Globecom Workshops (GC Wkshps), 2018, pp. 1-6.

[29] G. Yang, C. Li, and K. E. Marstein, "A blockchain-based architecture for securing electronic health record systems," Concurr. Comput. Pract. Exp., vol. 33, no. 14, p. e5479, 2021. 

[30] R. Widayanti, Q. Aini, H. Haryani, N. Lutfiani, and D. Apriliasari, "Decentralized Electronic Vote Based on Blockchain P2P," in 2021 9th International Conference on Cyber and IT Service Management (CITSM), 2021, pp. 1-7.

[31] G. Yang and C. Li, "A design of blockchain-based architecture for the security of electronic health record (EHR) systems," in 2018 IEEE International conference on cloud computing technology and science (CloudCom), 2018, pp. 261-265. 